\title{
Concluding Remarks II
}

\author{
Hubert Reeves
}

\section{Introduction}

The symposium has shown that our subject is well and very alive. It is progressing rapidly, thanks to a large amount of new observational data, obtained in particular by a young generation of competent astronomers. It is encouraging , incidentally, to note the large fraction of women in this generation. When I started in this field, some forty years ago, the feminine contribution was much smaller. I plan to review the state of the situation for each of the three nucleosynthesis processes responsible for the light elements: Big Bang Nucleosynthesis ( $\mathrm{BBN}$ ), galactic cosmic ray spallation of interstellar nuclei (GCR) and stellar nucleosynthesis. I will point out their successes, their riddles and possible avenues by which these riddles could be solved. I plan first to review the observations.

\section{Observatio Results}

\subsection{Deuterium}

We have three sets of historical data (fig 1), pertaining respectively to the present ISM (interstellar medium), the protosolar nebula (4.5 billion years ago) , and the early universe ( some 14 billion years ago). Linsky (1999), VidalMadjar, Ferlet and Lemoine (1997) , Vidal-Madjar (1999) and Sahu (1999) have presented and discussed the $\mathrm{D} / \mathrm{H}$ measurements in ISM , in front of bright stars - The estimated value of $\mathrm{D} / \mathrm{H}$ is $1.5 \times 10-5,(+/-0,10)$. Inhomogeneities of $\mathrm{D} / \mathrm{H}$ in the ISM are reported. The FUSE satellite, recently put into orbit, is expected to clear up this delicate point.

An argument based on the measurement of the helium isotopic ratio in the solar system can be used to give an estimate of the $\mathrm{D} / \mathrm{H}$ ratio in the galactic gas at the birth of the sun , 4,5 billion years ago (Geiss and Reeves 1972), on the assumption that the solar wind $3 \mathrm{He}$ is the sum of the protosolar $\mathrm{D}$ burned in $3 \mathrm{He}$ plus the protosolar 3He. In 1969 Johannes Geiss and his colleagues in Bern measured the $3 \mathrm{He} / 4 \mathrm{He}$ ratio in the solar wind from an aluminum sheet brought back from the moon (Geiss and Gloeckler 1997). Since the solar convective zone is hot enough to burn $\mathrm{D}+\mathrm{p}-\mathrm{i} 3 \mathrm{He}$, it was clear that the solar wind contains the sum of the primordial $\mathrm{D}$ and $3 \mathrm{He}$ of the protosolar nebula. But was it possible that, during the past history of this zonal material, some $3 \mathrm{He}$ had been burned in $4 \mathrm{He}$ ? The presence of $9 \mathrm{Be}$ in the solar photosphere at essentially the meteoritic ( i.e. protosolar value) argued against this possibility. In the surface convective zone of the sun, the destruction of the $9 \mathrm{Be}$ occurs at lower temperature than the $3 \mathrm{He}$ burning reactions. Hence any $3 \mathrm{He}$ depletion would have been accompanied by an even stronger $9 \mathrm{Be}$ depletion. This does not seem to 


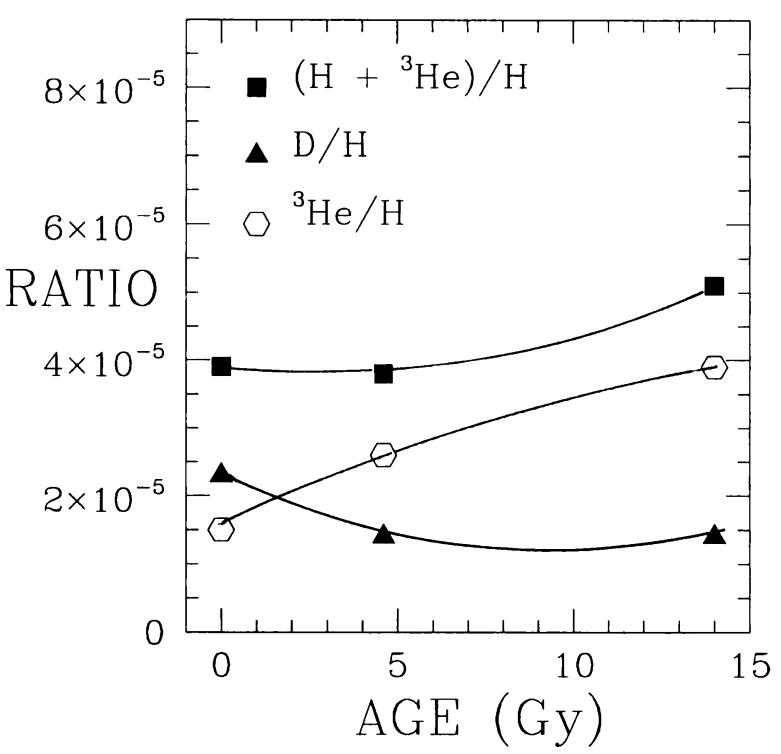

Figure 1. Deuteron and ${ }^{3} \mathrm{He}$ abundances as a function of time from BBN (14 Gy ago) till today ( adapted from Geiss and Gloeckler 1997). The value of ${ }^{3} \mathrm{He}$ at $14 \mathrm{~Gy}$ is not an observation but results from BBN calculations. The apparent variation of this nuclide at later time is well within the uncertainties.

be the case. The protosolar $3 \mathrm{He} / 4 \mathrm{He}$ needes to pursue this argument is obtained from the Jupiter value (discussed later). Subtracting it from the solar wind value one obtains a protosolar $\mathrm{D} / \mathrm{H}=1.9$ (+or-0,5)x10-5 (Gloeckler 99) . This is quite in agreement with the Jupiter $\mathrm{D} / \mathrm{H}$ value which have been reported by Donahue and Nieman (97) at $(2.6+/-0.7) \times 10-5$. Other jovian values (Gautier and Owen 1989), (Bjoraker et al 1986), (Carlson et al 1983), (Encrenaz et al 1996, ), range from 2 to $5 \times 10-5$. Because of possible chemical fractionations of hydrogen in the jovian atmosphrer, one cannot readily obtain the protosolar value from these measurements. Deuterium measurements by line absorpions on remote intergalactic clouds in front of quasars have led to a period of confusion - A high value $\mathrm{D} / \mathrm{H}$ of a few times 10-4 was first obtained by Songaila et al (1997) (discussed by Hogan 1997) A lower value of $(2,5(+/-0,2)$ x10-5) was later reported by Tytler (1997) . An analysis of the Tytler data by Levshakov et al (1997), (1999) give a $\mathrm{D} / \mathrm{H}$ value $4,5 \times 10-5$. There appears to be a consensus for such low values against the early high values. The possibility of spatial variations of deuterium in these absorbing clouds is discussed by Vidal-Madjar et al (1997) .Again the situation should cleared up with the recently launched FUSE satellite.

\subsection{Helium-3}

A measurement of $3 \mathrm{He} / 4 \mathrm{He}=2.48(+/-0,65) \times 10-4$ in the ISM near the solar system has been obtained by (Geiss and Gloeckler (1997) . Rood et al (1997) 
have measured $3 \mathrm{He} / 4 \mathrm{He}$ values in ionized hydrogen nebulae ( HII regions) with different oxygen abundances, situated at various galactocentric distances. These abundances are in approximate agreement with the Jupiter value : $3 \mathrm{He} / 4 \mathrm{He}=$ 1,03 (+or-0,06)× $10-4$ (Donahue and Nieman 1997) and with the Geiss and Gloeckler (1997) ISM value. With a dispersion of a factor three, there appears to be no systematic trends with galactocentric distance and with the abundance of elements other than hydrogen and helium ( called "metallicity" in the astronomer's jargon). Geiss and Gloeckler (1997) mention the small increase between the birth of the solar system and the present gas .

\subsection{Helium-4}

Helium-4 is generated in the Big Bang and also by Main Sequence stars. The observed fractional mass abundances range from 24the gradual effect of stellar energy generation in galaxies. The search for the primordial yield is conducted in objects which have been least contaminated by stellar synthesis, such as the extra galactic HII regions ( blue compact galaxies), as is evidenced by their comparatively low abundance of heavier isotopes (Peimbert1999). One strategy consists of a plot of $\mathrm{He}$ versus $\mathrm{O}$ or $\mathrm{C}$ or $\mathrm{N}$, which is tentatively extrapolated to zero value for these stellar-generated atoms. Another estimate is obtained by averaging on the objects with the lowest metal abundances. (Kunth and Sargent 1983) (Pagel 1989, 1992), Pagel et al 1992), (Campbell 1992), (Fuller et al 1992 ) (Thuan and Izotov 1997) (Thuan 1999) (Viegas 1999) . A primordial value of the helium mass fraction of $0,230-0.245$ is obtained. There is much debate regarding the uncertainties attached to this number.

\subsection{Lithium -7}

New measurements of its abundances in stars have been reported by (Pasquini 1999), (Hill 1999). (Kajino 1999),(Randich 1999), (de la Reza1999), (Malik1999), (Thevenin1999), (Torres1999). One of the best arguments in favor of a Big Bang contribution to the abundance of $7 \mathrm{Li}$ comes from the fact that, for old stars, $7 \mathrm{LI} / \mathrm{H} 1,5 \times 10-10$, is independent of the metallicity, for $\mathrm{Fe} / \mathrm{H}$ ratio varying from 10-3.5 to almost 10-1 solar (Spite and Spite 1982ab); (Spite et al 1997) (Hobbs and Duncan 1987), (Rebolo et al 1988, 1992). The "flatness" of the plateau is discussed by (Cayrel 1997, 1999) and (Spite 1997)( Molaro 1999).(Ryan 1999) To obtain the Big Bang yield from these observations one has to estimate the lithium depletion from surface processes in these old stars. This turns out to be a difficult problem with remaining unsolved features (Michaud and Charbonneau 1991) ( Vauclair 1999) (Michaud 1999). Nevertheless the flatness of the plateau (with a dispersion of less than a factor of two), suggest a rather small amount of surface stellar depletion, less than a factor of two, at best comparable to the dispersion.

\subsection{Beryllium-9}

This atom has been observed in a large number of young (Pop 1) stars (Boesgaard and Heacox1978),( Boesgaard and Ryan 1999) (Israelian1999). The mean value is in good agreement with the solar value $\mathrm{Be} / \mathrm{H}=1,4 \times 10-11$ measured by Chmielsvski et al 1975, Balachandran (1999) has shown than this value does not differ by more than 20carbonaceous chondrite) value (Grevesse and Noels 1992). 
In old (PopII) stars beryllium shows a systematic decrease with decreasing stellar metallicity (Gilmore et al 1991) (Gilmore et al 1992, Gilmore 1992) ; (Ryan et al1991) (Ryan 1992, 1999) Rebolo et al 1992) (Ryan 1999). The slope of the $\mathrm{Be} / \mathrm{H}$ vs $\mathrm{Fe} / \mathrm{H}$ indicates a linear growth of Be with $\mathrm{Fe}$ during the galactic life. Unlike Li however, Be shows no sign of a plateau at very small metallicity.

\subsection{Boron}

With an abundance ratio of $\mathrm{B} / \mathrm{Be}=20$ to 40 , the $\mathrm{B} / \mathrm{H}$ ratio behaves very much like the $\mathrm{Be} / \mathrm{H}$ ratio (Boesgaard and Heacox(1978) )(Primas et al 1999) (Primas 1999). It has recently shown (Cunha and Smith 1999) (Cunha 1999) that the solar value is in agreement with the meteoritic value $\mathrm{B} / \mathrm{H} 7 \times 10-10$, (Anders and Grevesse 1989), thereby solving a longstanding reported discrepancy between the two values. . With decreasing metallicity , $\mathrm{B} / \mathrm{H}$ decreases down to 10-12 with no sign of a plateau. . As in the case of $\mathrm{Be}$, the slope of the $\mathrm{B} / \mathrm{H}$ vs $\mathrm{Fe} / \mathrm{H}$ is close to one showing again a linear growth of $\mathrm{B}$ with $\mathrm{Fe}$ along the galactic life. The boron isotopic ratio in the solar system is $11 \mathrm{~B} / 10 \mathrm{~B}=4,050,05$. (Shima and Honda 1962) with variations of a few percent Chaussidon and Robert 1998 . Measurements of $11 \mathrm{~B} / 10 \mathrm{~B} 3.6$ have been made on two stars (Nissen 1999) . In the interstellar medium the situation appears to be confused. ( Rebull et al 1998)( Knauth 1999)

\section{Big Bang Nucleosynthesis}

The aim of the theory of Big Bang Nucleosynthesis is to account for the abundances of the nuclides $\mathrm{D}, 3 \mathrm{He}, 4 \mathrm{He}$ and $7 \mathrm{Li}$ in the very early universe, prior to the birth of galaxies and stars. (Schramm 1992) (Steigman 1999) (KurkiSuonio1999). Over the past three decades, BBN has progressively reached a high degree of credibility. By providing initial galactic abundances of these nuclides it has become a major tool for the study of stellar and galactic evolution. It also serves as a test-bench for new cosmological ideas. Big Bang nucleosynthesis has one free parameter : the baryonic number $h$ (the ratio of the number of nucleons to the number of photons) or equivalently $\mathrm{Wb}$ the ratio of the baryonic density to the critical density. The main success of $\mathrm{BBN}$ comes from the fact that with $\mathrm{h}=(5+/-1) \times 10-10$ corresponding to $\mathrm{Wb}=0.04+/-0.15 \mathrm{BBN}$ one can satisfactorily account for the primordial abundances of the three nuclides (D, $4 \mathrm{He}$ and $7 \mathrm{Li}$ ) while being compatible with $3 \mathrm{He}$ ( for which we have , however no primordial abundance measurement ; the value of $3 \mathrm{He} / \mathrm{H}$ at $\mathrm{BBN}$ in fig 1 has been computed with the value of $h=(5+/-1) \times 10-10)$ Let us call "cosmological window" the range of baryonic densities allowed by astronomical observations. The density Wlum of luminous matter ( stars and hot gases) is evaluated at 0.003 . This is clearly a lower limit to the universal nucleon density. On the other hand, the total matter density Wmat, estimated by its gravitational manifestations ( in Einstein theory, all forms of matter and energy exert gravitational effects), is close to 0.3 ; an upper limit to $\mathrm{Wb}$. Thus the $\mathrm{BBN}$ calculated $\mathrm{Wb}$ manages to account for the abundances of the primordial nuclides while being compatible with the cosmological window $(0.003 ; \mathrm{Wb} ; 0.3)$ This coherence between the nuclear physics and astronomical domains is another strong argument in favor of the Big Bang theory. An evaluation of Wb can also 
be obtained from the analysis of the absorption lines in the spectra of remote quasars. These lines are ascribed to diffuse clouds of matter in intergalactic space. The computations, although somewhat model-dependant, yield indeed values of $\mathrm{Wb}$ compatible with the $\mathrm{BBN}$ value. One last point before we close the subject of the Big Bang. Recent experiments (Normile 1998), have given a mass of approximately $0.1 \mathrm{eV}$ for one of the three varieties of neutrinos ,(probably the tau neutrino). The others varieties are most likely even less massive. This insures that all three neutrinos are relativistic at $\mathrm{BBN}$ ( $\mathrm{kT}=$ one $\mathrm{Mev}$ ) , and thus justifies the standard model for which the choice of three relativistic neutrinos. This was previously an assumption.

\section{BBN as a tool for stellar and galactic evolution}

\subsection{Deuterium}

During galactic evolution,deuterium is systematically destroyed by astration, thus we expect a gradual depletion of its BBN abundance as a function of time , as more galactic gas is incorporated in stars and later ejected with $\mathrm{D}$ free gas - This depletion is plausibly tempered by the continuous infall of extragalactic gas on the galactic disk, with presumably pristine D (Lubovich 1999) . Several authors (Tosi 1997), (Prantzos et al 1992), (Prantzos 1996), (Matteuci 1999 ), (Olive 1999), (Chiappini 1999), (Vangioni-Flam, Olive and Prantzos 1994) (Olive 1999) have presented consistent galactic evolution models incorporating relevant astronomical observations such as the abundance ratios of heavier elements, the galactic mass fractions of stars and gaseous nebulae, as a function of age and radial distance from the center of the galaxy. These evolution models include up-to-date nuclear and atomic data for the various elements and isotopes of interest. The initial input of these models are 1) the rate of star formation 2) the Initial Mass Function and 3) the rate of infall of extragalactic matter. In this respect the observations of (Beers 1999) on the population of very metal poor stars are of great interest. The general conclusion is that models which accounts satisfactorily for the ensemble of observations generate only a modest amount of $\mathrm{D}$ depletion - at best by a factor of three- , in good agreement with the data (fig1).

\subsection{Helium-3}

This nucleus is produced in significant quantity both by BBN and by stellar synthesis, as an intermediate step in hydrogen burning. In stars, 3He is produced during the Main Sequence phase by the $(D+p)$ reaction. In the hot centers , $3 \mathrm{He}$ is further transformed in $4 \mathrm{He}$ by the $3 \mathrm{He}+3 \mathrm{He}$ or $3 \mathrm{He}+4 \mathrm{He}$ reactions . In the outer stellar layers however the temperature is high enough to generate $3 \mathrm{He}$ but not to destroy it. Stellar models show there an accumulation of $3 \mathrm{He}$ reaching values of $3 \mathrm{He} / 4 \mathrm{He} 10-2$ As the star moves toward the red giant branch, a fraction of these nuclei is burned into $4 \mathrm{He}$ while another fraction is convected to the surface and ejected by stellar winds. Other processes in novae or other advanced stages of stellar evolution may further generate significant amounts of $3 \mathrm{He}$. The final fate of an $3 \mathrm{He}$ is governed by stellar internal processes, from the Main Sequence to the Giant Branch and the Planetary Nebula phases, through 
the effects of various dredge-up and mixings. The conventional stellar models predict an important yield of $3 \mathrm{He}$ for stars smaller than 2 solar masses. Observations of Planetary Nebulae (PN) (Rood et al 1997 ) have confirmed this predictions with some detections at the level of $3 \mathrm{He} / 4 \mathrm{He} 10-3$. The problem lies with the theoretically expected stellar contribution of these PN during the life of the galaxy. According to conventional models, values of $3 \mathrm{He} / 4 \mathrm{He} 10-3$ should be observed in the interstellar medium, quite in disagreement with the measurements. Charbonnel et al (1997), Charbonnel (1999) have discussed the relation between this problem and the problems raised by the observations of $12 \mathrm{C} / 13 \mathrm{C}$ ratio and of $\mathrm{N}$ and $\mathrm{O}$ abundances in $\mathrm{PN}$. The discrepancy could be solved by the operation of a new mixing phase in the Red Giant Branch, which would account for the destruction of $3 \mathrm{He}$ and also for the unexpectedly low $12 \mathrm{C} / 13 \mathrm{C}$ observed in some $\mathrm{PN}$. The large scatter in the observed abundances of $3 \mathrm{He}$ makes it difficult to estimate the relative contributions of $\mathrm{BBN}$ and stellar evolution to its abundance. The $\mathrm{BBN}$ value, shown in fig 1 , has been computed in the frame of BBN using the baryonic number obtained from $\mathrm{D}, 4 \mathrm{He}$ and $7 \mathrm{Li}$. Helium-4 After the BBN production of an helium fractional mass of 0,24 , the main effect of all the Main Sequence stars has been to increase its galactic value to 0,30 implying a transformation of $64 \mathrm{He}$ corresponding to an energy release of $0,4 \mathrm{MeV}$ per nucleon.

\subsection{Lithium-7}

The BBN contribution to $7 \mathrm{Li}$ dominates the galactic gas abundance in the first billions of years. The flatness of the curve however has far reaching implications for the hydrodynamics of stellar surface layers. The effects of atomic (microscopic) diffusion below the convective zone should results in lithium abundances varying with the mass and surface temperature of the star in which they are observed (contrary to observations) unless these effects are neutralized by others phenomena. These as been a problem for many years. Several groups (Delyannis 99) (Pinsonneault et al 1992, Pinsonneault 1999, Vauclair 99) have studied the possible influence of various physical phenomena such as mixing via differential rotations, gravity waves and stellar winds. In all cases the flatness of the curve imposes important constraints on the efficiency of these processes. The flatness of the lithium abundance curve as a function of metallicity argues against heavy stellar astration and hence against important $\mathrm{D}$ depletion for metallicity less than one tenth solar. When the galactic mass fraction of heavy elements became larger than one part in a thousand (one tenth the solar value) a new stellar source (Sackman 1999) managed to increase the abundance of lithium by an extra factor of ten. The observations of lithium abundances in the surface of evolved stars of the Asymptotic Giant Branch (AGB stars) at a value appreciably larger than PopI value of $2 \times 10-9$ suggest that these stars have contributed, by mass ejection, to this enrichment of galactic lithium . Other candidates such as novae and supernovae have also been suggested. Convincing quantitative models of the galactic enrichment are still lacking. The GCR production of $7 \mathrm{Li}$, evaluated through the abundance of $9 \mathrm{Be}$, never dominates the abundance curve.

Lithium has been observed in a large number of stars in various states of evolution. The study of the abundance variations is a very important tool for the elucidation of the physics of stars and galactic evolution. 


\section{GCR origin of $\mathrm{LI} \mathrm{Be} \mathrm{B}$}

We consider first the case of $9 \mathrm{Be}$. It is a typical case of a nucleus for which we know of no low energy production mechanism. We have to resort to spallation reactions in interstellar space. The rate of formation of $9 \mathrm{Be}$ by GCR is given by the product of the flux of high energy protons $(16 \mathrm{~cm}-2 \mathrm{sec}-1)$ times the crosssections for $9 \mathrm{Be}$ formation by proton collision on the most abundant targets $16 \mathrm{O}$ and $12 \mathrm{C}(5 \mathrm{mb})$ times the abundance ratio of these targets to hydrogen (10-3) in space. As mentioned by Parizot 1999 in his review talk, the approximate equality between the product of the formation rate times the age of the galaxy, on the one hand, and the beryllium to hydrogen ratio in recent stars (10-11) is the best evidence for a major GCR contribution to $9 \mathrm{Be}$ and by the same token to some of the light elements of the group Li Be B (Reeves et al 1970). To pursue this idea, the important parameters are the excitation functions for spallation reactions induced by protons and alphas. In principle all the nuclei with $\mathrm{A}_{i} 11$ in interstellar space are target candidates. In practice, only $16 \mathrm{O}, 12 \mathrm{C}$ and marginally $14 \mathrm{~N}$ are abundant enough to contribute appreciably. Alpha + alpha reactions may have been important in the early days of the galaxy (Montmerle 1977)(Steigman and Walker 1992). Thanks mostly to the pioneering work at the Orsay laboratory in France, the important excitation functions are now known with sufficient accuracy ( (reviewed in Reeves 1974 and Read and Viola 1985) . The number of thermalized nuclides added to the interstellar gas per unit time is the sum of two contributions. First : spallation of interstellar heavy nuclei by fast protons and alphas. In this case, the recoil energy of the spallation products is small (a few $\mathrm{MeV}$ per nucleon) and they suffer no further destruction or loss. Second: spallation of fast heavy nuclei by interstellar $\mathrm{H}$ and $\mathrm{He}$. The spallation products are partially destroyed during their deceleration by electron collisions. To compare theory and observations, it is convenient to study separately two phases of the galactic life. First : the "recent" era (the last ten billion years or so, corresponding more or less to PopI stars) and second: the early days of the galaxy (PopII stars).

\section{The recent galactic era}

Comparing the ratios of the spallation cross-sections of protons on $\mathrm{O}$ and $\mathrm{C}$ to the ratios of the stellar abundances of $\mathrm{Li} \mathrm{Be} \mathrm{B}$ confirms the view that some of the light nuclei are generated by GCR. The analysis, shows that the GCR mechanism satisfactorily accounts for $6 \mathrm{Li}, 9 \mathrm{Be}, 10 \mathrm{~B}$, gives a major contribution to the abundance of 11B and a minor (10abundance of 7Li (Reeves et al 1970)(Meneguzzi et al 1971)(Reeves et al 1973) Another source of $7 \mathrm{Li}$ is clearly required. A smaller discrepancy exists also for the boron isotopic ratio which will be discussed separately. The early galactic era. The study of the GCR generated elements in PopII stars may yield important clues to the physical conditions accompanying the formation of galaxies. (Ryan et al 1991,1992 ) (Rebolo et al 1988; 1992) ; ( Spite et al 1991); (Duncan 1997) ; (Gilmore et al 1991), (Smith et al 1992).(Primas1999) (Cunha 1999). The growth of the $\mathrm{Be} / \mathrm{H}$ and $\mathrm{B} / \mathrm{H}$ as a function of the metallicity $(\mathrm{Fe} / \mathrm{H})$ of the galaxy appears to be linear. The implications of this behaviour are still under discussions. This 
result suggests that the ratio of fast $(\mathrm{CNO} / \mathrm{HHe})$ was much larger in the early GCR than today (Cass 1999) (Ramaty 1999) (Parizot 1999). In the astrophysical context, this situation could occur in the "superbubble scenario". Regions of massive star formation ( $\mathrm{OB}$ associations, starbursts), containing numerous supernovae ejecta enriched in heavy elements, are assumed to be seat of intense shock wave acceleration processes.

\section{The isotopic composition of lithium}

Four cases of stellar 6Li idenfifications in old Pop II stars are reported, all at the levels of 5 the observational lower limit. Considering the fact that $6 \mathrm{Li}$ is far more fragile than $7 \mathrm{Li}$, these detections are often presented as a strong argument for the case of no large depletion of $7 \mathrm{Li}$ in those stars. One point however remains worrysome. Given that the theoretically possible range $6 \mathrm{Li} / 7 \mathrm{Li}$ extends almost to 100 all four values are at the lower observational limit is somewhat suspicious. Systematic uncertainties may be larger than expected. Finding even one other Pop II star with an higher value of $6 \mathrm{Li} / 7 \mathrm{Li}$ would ease the situation. If confirmed , the detection of $6 \mathrm{Li}$ in metal poor stars would provide the first evidence for the contribution of $\mathrm{a}+\mathrm{a}$ reactions to the abundance of $\mathrm{Li}$. It would also show that the GCR contributed a few percent to the Pop II lithium abundance.

Inhomogeneities in the $6 \mathrm{Li} / 7 \mathrm{Li}$ ratios in the present interstellar medium ranging from 0.08 ( the protosolar value) to almost 0.5 have been reported by Lemoine et al (1992), Federman (1999), (Knauth 1999), in regions of star formation ( Ophiucus and Persei ). The effect of strong irradiation of the interstellar medium by fast particles- producing local increase of $6 \mathrm{Li}$ - have been suggested, supported by observations of locally increased ionization rates (by a factor of ten over the mean interstellar value) (Federman 1999). However to produce a noticeable increase of $6 \mathrm{Li}$ would require a time- integrated flux comparable to the galactic time integrated flux (1010y). With (only) a factor ten increase in the ionization rate, the duration of this extra flux would have to last one GY (109y ), far longer than the duration of a star forming process ( $107 \mathrm{y})$. Local variations of $7 \mathrm{Li}$ could come from recent release of stellar-made $7 \mathrm{Li}$ by evolved stars. In this case the low protosolar 6/7 ratio (0.08) (implying a comparatively strong local contamination in $7 \mathrm{Li}$ ) would be difficult to reconcile with the fact that the protosolar $7 \mathrm{Li} / \mathrm{H}$ is in good agreement with the observed values found in young stars (implying no strong solar inhomogeneities). In summary the interpretation of these local lithium isotopic inhomogeneities poses a serious challenge to theorist!

\section{The isotopic composition of boron}

One long standing problem is the difference between the protosolar boron isotopic ratio $(11 \mathrm{~B} / 10 \mathrm{~B}=4.0$ (+or- 0.1$)$ and the value 2.5 generated by the Galactic Cosmic Rays, with mean energy around one $\mathrm{GeV}$. Given the shape of the spallation cross-sections leading to these isotopes at low energy it was proposed that the contribution of lower energy particles, unobservable from the inner solar system, could account for the difference (Reeves and Meyer 1978)(Ramaty et al 1999). Another solution exist : the neutrino disintegration of $12 \mathrm{C}$ which would 
generate far more $11 \mathrm{~B}$ than $10 \mathrm{~B}$. The process would take place in exploding stars (supernovae) in which intense fluxes of neutrinos emitted from collapsing cores (Woosley 1990) are later reabsorbed by the outer layers, thereby expelling them in the form of remnants. More isotopic boron ratio measurements in stars and in ISM are needed to decide if a neutrino contribution is mandatorily requires. Notice that, contrary to the case of the lithium isotiopic ratio, we do not expect differential depletion of $B$ on the Main-Sequence (except at the very cold end).

\section{Stellar nucleosynthesis}

Stellar contributions are required for $7 \mathrm{Li}$ and perhaps also for $11 \mathrm{~B}$. The existence of lithium rich stars ( more than the present cosmic abundanes $7 \mathrm{Li} / \mathrm{H} i 3 \mathrm{X} 10-$ 9) such as WZ Cassiopea and others, give is a strong motivation to the idea that stellar winds from such stars could have enriched the ISM with respect to the BBN value. The observations from (Castilho 1999) of giant stars with abundant lithium together with depleted Be and B confirms the occurence of lithum production mechanisms in advanced stages of stellar evolution. The formation mechanisms in stars of the Red Giant Branch, Asymptotic Giant Branch, novae , and supernovae avec been discussed by (Sackman 1999) and (Mateucci 1999 ) A report of infrared observations, from 25 to 60 microns, of lithium rich giants has been presented by de la Reza ( 1999). This study give access to shell ejections, the very mechanism by which the lithium atoms are most likely expelled and spread in the ISM.

\section{References}

Asplund, M., 1999 IAU Symposium 198 Natal , Brazil nov 1999

Anders, E., and N. Grevesse, 1989 Geochim. Cosmochim.Acta , 53, 197

Balachandran, S.C. ,Bell,R.A., 1998 Nature 392791 .

Beers, T.C., 1999 IAU Symposium 198 Natal , Brazil nov 1999

Bjoraker, G. et al 1986 Icarus, 66579.

Boesgaard, A.M.,and W.D. Heacox, 1978,Astrophys. J. 226888

Boesgaard, A.M., and Ryan S.G., 1999 IAU Symposium 198 Natal , Bra

Campbell, A., Astrophys. J. 1992401157

Carlson, B.et al 1993 J.Geophys.Rev. 98, 5251.

Cass , M., 1999 IAU Symposium 198 Natal , Brazil nov 1999

Castilho, B.V. 1999 IAU Symposium 198 Natal , Brazil nov 1999

Cayrel, R 1998, ISSI Workshop on Primordial Nuclei and Their Galactic Evolution, Space Science Reviews 84, N. Prantzos, M, Tosi and R.von Steiger editors (Kluwer Academic Press).

Cayrel, R. 1999 IAU Symposium 198 Natal , Brazil nov 1999

Charbonnel,C et al 1997 ISSI Workshop on Primordial Nuclei and Their Galactic Evolution. Berne Switzerland 6-10 May 1999, Space Science Review 
Kluwer Academic Press May 1997 N. Prantzos, M, Tosi and R.von Steiger editors .

Charbonnel, C., 1999 IAU Symposium 198 Natal , Brazil nov 1999

Chaussudon, M. et Robert, F 1998 Earth Planet. Sci. Lett. 164, 577

Chiappini, C., 1999 IAU Symposium 198 Natal , Brazil nov 1999

Chmielevski, Y., Muller, F.H., and Brault, J.W. 1975 Astron. Astrophys. 42, 37

Cunha, K. and Smith, V, ApJ.1999 5121006

Cunha , K., 1999 IAU Symposium 198 Natal , Brazil nov 1999

de la Reza , R., 1999 IAU Symposium 198 Natal , Brazil nov 1999

Delyannis, C., 1999 IAU Symposium 198 Natal , Brazil nov 1999

Donahue, T.M. and Nieman, H.B. 1998, ISSI Workshop on Primordial Nuclei and Their Galactic Evolution, Space Science Reviews 84, N. Prantzos, M, Tosi and R.von Steiger editors (Kluwer Academic Press).

Duncan, D 1998, ISSI Workshop on Primordial Nuclei and Their Galactic Evolution, Space Science Reviews 84, N. Prantzos, M, Tosi and R.von Steiger editors (Kluwer Academic Press).

Encrenaz, Th . et al., 1996 , A -A 315, L397

Federman 1999 IAU Symposium 198 Natal, Brazil nov 1999

Fuller, G.M., R.N. Boyd, and J.D. Kalen, 1991, ApJ. 371, L11 et al 1992

Gautier ,D., and T. Owen, 1989, in Origin and Evolution of atmospheres,

S.K. Atreya et al eds.( University of Arizona Press). p. 487

Geiss, J., P. Eberhardt, F. Buhler, J. Meister, and P.Signer, 1970, J. Geophys. Res. 75, 5972. a Geiss, J., and H. Reeves,1972, Astron. Astrophys. 18, 126 ,

Geiss, J . and Gloecker, G 1998, ISSI Workshop on Primordial Nuclei and Their Galactic Evolution, Space Science Reviews 84, N. Prantzos, M, Tosi and R.von Steiger editors (Kluwer Academic Press).

Gilmore, G., B. Edvardsson, and P.E. Nissen, 1991, Astrophys. J. 378, 17.

Gilmore, G., B. Gustafsson, B. Edvardsson, and P. Nissen, 1992, Nature 357, 379.

Gilmore, G., Symposium in "On the Origin and Evolution of the elements", Paris, June 1992, Prantzos, N., E. Flam, and M.Cass,. (Cambridge University Press).

Gloeckler, G. 1999 IAU Symposium 198 Natal , Brazil nov 1999

Grevesse, N and Noels,A 1992 in "On the Origin and Evolution of the elements", Paris, June 1992, Prantzos, N., E. Flam, and M.Cass,. (Cambridge University Press).

Hill, V., 1999. IAU Symposium 198 Natal , Brazil nov 1999

Hobbs, L.M., and D.K.Duncan,1987, Astrophys. J. 317, 796. Hogan , C.J. 1998, ISSI Workshop on Primordial Nuclei and Their Galactic Evolution, Space Science Reviews 84, N. Prantzos, M, Tosi and R.von Steiger editors (Kluwer Academic Press).

Israelian G., 1999 IAU Symposium 198 Natal , Bra 
Kajino ,T., Knauth,D 1999 IAU Symposium 198 Natal , Brazil nov 1999

Knauth,D 1999 IAU Symposium 198 Natal , Brazil nov 1999

Kunth, D., and W. Sargent, 1983, Astrophys. J. 273, 81

Kurki-Suonio, 1999 IAU Symposium 198 Natal , Bra

Lemoine, M., R. Ferlet, A. Vidal-Madjar, C. Emerich, P. Bertin, 1992, to appear in Astron. Astrophys. also in "On the Origin and Evolution of the elements", Paris, June 1992, Prantzos, N., E. Flam, and M.Cass, (Cambridge University Press).

Levshakov, S.A., et al 1998, ISSI Workshop on Primordial Nuclei and Their Galactic Evolution, Space Science Reviews 84, N. Prantzos, M, Tosi and R.von Steiger editors (Kluwer Academic Press).

Levshakov. S., 1999 IAU Symposium 198 Natal , Brazil nov 1999

Linsky, L.J. 1998, ISSI Workshop on Primordial Nuclei and Their Galactic Evolution, Space Science Reviews 84, N. Prantzos, M, Tosi and R.von Steiger editors (Kluwer Academic Press).

Linsky, J. 1999 IAU Symposium 198 Natal , Brazil nov 1999

Lubovich, D., 1999 IAU Symposium 198 Natal , Brazil nov 1999

Mallik,S.V., 1999 IAU Symposium 198 Natal , Bra

Matteucci, F 1999 IAU Symposium 198 Natal , Brazil nov 1999

Meneguzzi, M., J. Audouze, and H. Reeves, 1971, Astron. Astrophys., 15, 337,

Meneguzzi, M., and H . Reeves, Astron. Astrophys., 1975, 40, 91

Michaud, G., and P . Charbonneau, 1991, Space Science Reviews, 57, 1

Michaud, G.1999 IAU Symposium 198 Natal , Brazil nov 1999

Molaro., P. 1999 IAU Symposium 198 Natal , Brazil nov 1999

Montmerle , T., 197,7 Astrophys. J., 217, 872 .

Nakano, J., and K. Sato, Contributed paper of Annual Meeting of Phys. Soc. Japan ,1990, Oct.

Nissen,P.E. 1999 IAU Symposium 198 Natal , Brazil nov 1999

Normile, D., 1998 Science. 2801690.

Olive, K., 1999 IAU Symposium 198 Natal , Brazil nov 1999

Pagel , B. E. J., and E. A. Simonson, 1989, Rev. Mex. Astr. Astrophys. ,18, 153

Pagel , B. E. J., and E. A. Simonson, R. J. Terlevich, and M. G. Edmunds, 1992, Mon.Not. R. Astr. Soc., 255, 325.

Pagel, B. E. J ,1992, in " On the Origin and Evolution of the elements", Paris, June 1992, Prantzos, N., E. Flam, and M. Cass, (Cambridge University Press).

Parizot, E 1999 IAU Symposium 198 Natal , Brazil nov 1999

Pasquini, I, 1999 IAU Symposium 198 Natal , Brazil nov 1999

Peimbert, M., 1999 IAU Symposium 198 Natal , Brazil nov 1999

Pinsonneault, M. H., C.P. Delyannis, and P. Demarque, 1992, Astrophys. J., Supp 78, 179.

Pinsonneault, M.H. 1999 IAU Symposium 198 Natal , Brazil nov 1999 
Prantzos, N., 1998, ISSI Workshop on Primordial Nuclei and Their Galactic Evolution, Space Science Reviews 84, N. Prantzos, M, Tosi and R.von Steiger editors (Kluwer Academic Press).

Prantzos, N., M. Cass, and E. Viangoni-Flamm, Symposium in "On the Origin and Evolution of the elements", Paris, June 1992, Prantzos, N., E. Flam, and M.Cass,. (Cambridge University Press).

Primas,F.,Duncan,D.K.,Peterson,R.C.and Thoburn, J.A.1999 A\& A 343, 545

Primas,F.1999 IAU Symposium 198 Natal , Brazil nov 1999

Ramaty, R., Koslovsky, B., Lingenfelter,R.E and Reeves, H 1997, ApJ ,488, 730.

Ramaty,R., 1999 IAU Symposium 198 Natal , Brazil nov 1999

Read, S. M., and V. E. Jr Viola, 1984, . At. Data . Nucl. Tables, 31,359.

Rebolo, R., P. Molaro, and J. E. Beckman, 1988, Astron. Astrophys. ,192, 192.

Rebolo, R., 1990,Proceedings of the Cambridge Conference on Elements in the cosmos

Rebolo, R., R. J. Garcia-Lopez, E. L. Martin, J. E. Beckman, C. D. McKeith, J.K. Webb, and Y.V. Pavlenko, 1992, in "On the Origin and Evolution of the elements", Paris, June 1992, Prantzos, N., E. Flam, and M.Cass, (Cambridge University Press).

Reeves, H., W. A. Fowler, and F. Hoyle, 1970, Nature (London), 226, 727

Reeves, H., J. Audouze, W.A. Fowler, and D.N. Schramm, 1973, Astrophys. J. , 177, 909

Reeves, H., 1974, Ann. Rev. Astr. Ap, 12, 437.

Reeves, H., and J.P. Meyer, 1978, Astrophys. J., 226, 613.

Rood, R. T., T. M. Bania, and T. L. Wilson, 1992, Nature (London), 355, feb 13

Rood, R.T. et al 1998, ISSI Workshop on Primordial Nuclei and Their Galactic Evolution, Space Science Reviews 84, N. Prantzos, M, Tosi and R.von Steiger editors (Kluwer Academic Press).

Rood, R.T. and Bania, T.M. 1999 IAU Symposium 198 Natal , Brazil nov 1999

Ryan, S. G., J. E. Norris, and M. S. Bessel, 1991, Astron. J., 102, 303.

Ryan, S. G., in "On the Origin and Evolution of the elements", Paris, June 1992, Prantzos, N., E. Flam, and M.Cass, (Cambridge University Press).

Ryan,S.G. 1999 IAU Symposium 198 Natal , Brazil nov 1999

Sackman, J., 1999 IAU Symposium 198 Natal , Brazil nov 1999

Sahu, K., 1999 IAU Symposium 198 Natal , Brazil nov 1999

Schramm, D. N., 1992, in " On the Origin and Evolution of the elements", Paris, June 1992, Prantzos, N., E. Flam, and M.Cass, (Cambridge University Press).

Smith , V. V., D. L. Lambert, and P. E. Nissen, 1992, "The $7 / 6$ ratio in two metal poor stars", Astron.Astrophys.J. 408, 262 Astrophys. J. , sept 1992

Songaila, A. et al, 1997, Nature, 385, 137.

Spite, M., and F. Spite, 1982a, Nature (London), 297, 483.

Spite, F., and M. Spite,1982b, Astron. Astrophys., 115, 337. 
Spite et al 1998, ISSI Workshop on Primordial Nuclei and Their Galactic Evolution, Space Science Reviews 84, N. Prantzos, M, Tosi and R.von Steiger editors (Kluwer Academic Press).

Spite, F 1999 IAU Symposium 198 Natal , Brazil nov 1999

Steigman, G., and T. P. Walker, 1992, Astrophys. J., Lett., 385, L13.

Steigman, G., and M.Tosi, 1992, Astrophys. J. ,401, 150.

Steigman, G. 1999 IAU Symposium 198 Natal , Brazil nov 1999

Thevenin,F 1999 IAU Symposium 198 Natal, Brazil nov 1999

Thuan, T,X and Isotov, Y, I 1998, ISSI Workshop on Primordial Nuclei and Their Galactic Evolution, Space Science Reviews 84, N. Prantzos, M, Tosi and R.von Steiger editors (Kluwer Academic Press).

Thuan, T.X., 1999 IAU Symposium 198 Natal, Brazil nov 1999

Torres, C.A.O.,1999 IAU Symposium 198 Natal , Bra

Tosi, M., 1998, ISSI Workshop on Primordial Nuclei and Their Galactic Evolution, Space Science Reviews 84, N. Prantzos, M, Tosi and R.von Steiger editors (Kluwer Academic Press).

Tosi , M., 1999 IAU Symposium 198 Natal , Brazil nov 1999

Tytler, D. 1998, ISSI Workshop on Primordial Nuclei and Their Galactic Evolution, Space Science Reviews 84, N. Prantzos, M, Tosi and R.von Steiger editors (Kluwer Academic Press).

Tytler, D 1999 IAU Symposium 198 Natal, Brazil nov 1999

Vangioni-Flam, E., Olive, K. and Prantzos, N. 1994, ApJ, 148, 3. Vauclair, S., and Charbonnel, C., 1998 ApJ 502, 372,

Vauclair, S., 1999 IAU Symposium 198 Natal , Brazil nov 1999

Viegas, S; 1999 IAU Symposium 198 Natal , Brazil nov 1999

Vidal-Madjar, A Ferlet, R and Lemoine , M 1998, ISSI Workshop on Primordial Nuclei and Their Galactic Evolution, Space Science Reviews 84, N. Prantzos, M, Tosi and R.von Steiger editors (Kluwer Academic Press).

Vidal-Madjar., A., 1999 IAU Symposium 198 Natal , Brazil nov 1999

Walker, T.P., G.J. Mathews, and V.E. Viola, 1985, Astrophys. J. , 299, 745

Woosley, S.E., D.H. Hartman, R.D. Hoffman, and W.C. Haxton, 1990, Astrophys. J., 356, 272. 